\title{
Quantitative Electron Diffraction of Graphene: Measurement of Mean-Square Atomic Displacement and Implications for Dark Field Imaging.
}

\author{
Matthew Mecklenburg ${ }^{1,2,3}$, Brian Shevitski ${ }^{1,2}$, William Hubbard ${ }^{1,2}$, E. R. White ${ }^{1,2}$, Ben Dawson ${ }^{4}$, M. S. \\ Lodge $^{4}$, Masa Ishigami ${ }^{4}$, and B. C. Regan ${ }^{1,2}$ \\ 1. Department of Physics and Astronomy, University of California, Los Angeles, California 90095, USA \\ 2. California NanoSystems Institute, University of California, Los Angeles, California 90095, USA \\ 3. Microelectronics Technology Department, The Aerospace Corporation, Los Angeles, California \\ 90009, USA \\ 4. Department of Physics and Nanoscience Technology Center, University of Central Florida, Orlando, \\ Florida 32816, USA
}

Imaging and diffraction by transmission electron microscopy (TEM) can be used to measure atomic positions in solids. TEM can detect the mean-square displacement of atoms from their lattice positions, and the study of these displacements is relevant to high resolution transmission electron microscopy (HRTEM) image simulation [1], temperature measurements with sub-optical wavelength spatial resolution, and the melting temperature of solids. Because graphene is atomically thin it scatters relativistic electrons without dynamic scattering effects, and is therefore a useful material for precisely studying atomic positions. Using the simpler kinematic scattering approximation, we can describe the diffraction pattern using an analytic expression. By fitting our analytical expression to experimentally acquired diffraction patterns we measure the mean-square displacement of atoms from their ideal lattice positions in graphene [2].

We acquired diffraction patterns of our samples in an FEI Titan 80-300 transmission electron microscope and recorded the images using a Gatan Ultrascan CCD camera. One such diffraction pattern is shown in Figure 1a. The cartoons in Figures $1 \mathrm{~b}$ and $1 \mathrm{~d}$ reveal the connection between the relative atomic positions in graphene and the structure factor, which is illustrated in the intensities of the diffraction peaks. By carefully aligning the microscope and using acquisition times of a few seconds we were able to detect more than 19 diffraction orders. Fitting the peak intensities as a function of scattering angle enabled us to measure the peaks' damping caused by atomic motions. The fit parameters are: an amplitude proportional to the beam current (unmeasured), the screening length of the Yukawa potential used to model the atom, and the mean-square displacement of the carbon atoms. The screening length was measured to be $34 \pm 2 \mathrm{pm}$, which is in reasonable agreement with the ThomasFermi screening length of $29 \mathrm{pm}$, and in good agreement with a relativistic Hartree-Fock calculation of $33 \mathrm{pm}$. The mean-square displacement was measured to be $40 \pm 10 \mathrm{pm}^{2}$, which is consistent with an estimate based on the Debye phonon model [3].

Once a full understanding of single layer graphene diffraction is complete, a generalization to multilayer graphene is straightforward. With this, we can understand why second order peaks are preferable to first order peaks when using dark field imaging to highlight grain boundaries. Figure 1d-e shows the second order peaks should be more intense than the first order peaks. First and second order peaks have roughly the same intensity in ideal monolayer graphene. However, the second order peaks have much less background (either from thermal diffuse scattering or scattering from an amorphous support), and therefore make dark field images with better contrast. Figure 1 also illustrates that the first order peaks do not change intensity between single-layer graphene and bilayer graphene, unlike the second order 
peaks. Thus the second order peaks can be used to distinguish single and bilayer graphene, as shown in Figure 2.

We have found that the diffraction pattern of graphene can be modeled analytically. Fitting our analytic model to measured diffraction intensities allows us to measure the picometer scale mean-square displacements atoms. This measurement is useful for better modeling of HRTEM images, understanding nano-scale temperature gradients, and understanding the melting point of solids. In addition we have shown that a quantitative understanding of electron diffraction from graphene is useful for a better understanding of dark field imaging of graphene. The work at The Aerospace Corporation was supported under its Independent Research and Development Program.

References:

[1] A Howie, Hunting the Stobbs factor, Ultramicroscopy, 98 (2004), p. 73.

[2] B Shevitski et al, Dark-field transmission electron microscopy and the Debye-Waller factor of graphene, Phys. Rev. B, 87 (2012), p. 045417.

[3] G Mahan, "Many Particle Physics (Physics of Solids and Liquids)", Springer; 3nd edition (January $15,2000)$.
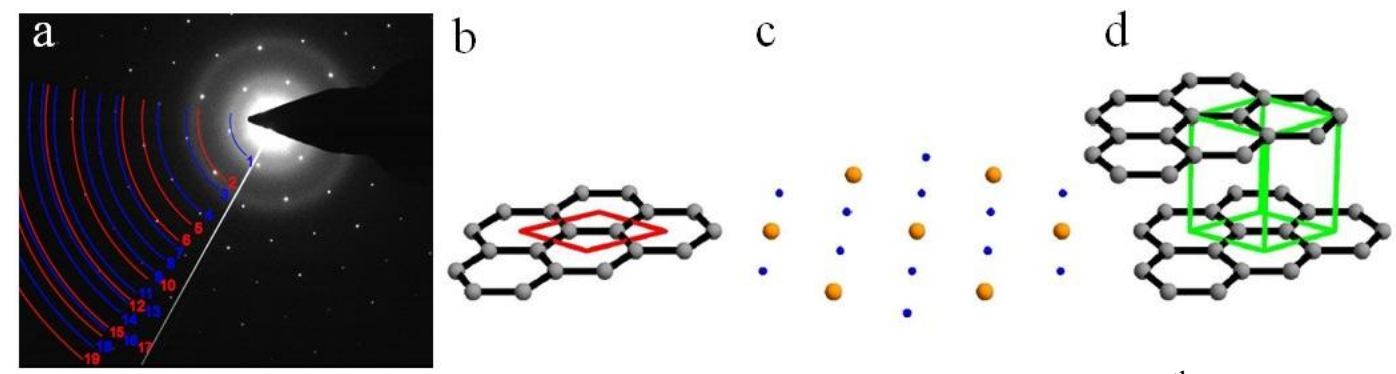

e

Figure 1. (a) A measured diffraction pattern of graphene out to $19^{\text {th }}$ order. (b) Single layer graphene with its unit cell outlined in red. (c) A reciprocal space schematic of graphene. Blue (orange) dots have a squared structure factor value of 1 (4). (d) A section of bilayer graphene with its unit cell outlined in green. (e) A reciprocal space schematic of bilayer graphene with the blue (orange) dots having a squared structure factor value of 1 (16).

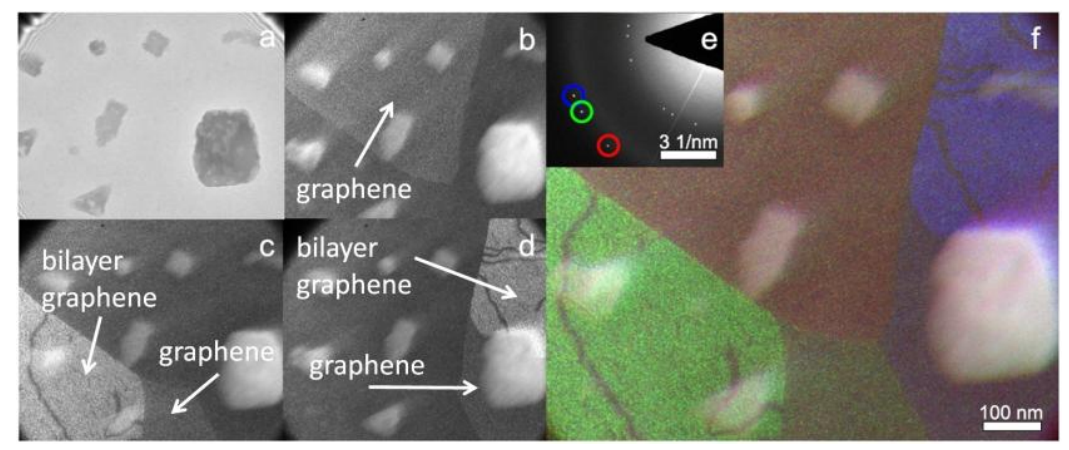

Figure 2. (a) Bright field image of graphene containing left-over processing material on the surface. (b)-(d) Dark field images of the same region. Each image has a different crystal direction highlighted and the second order diffraction peaks representing each are indicated in the diffraction pattern in (e). Using second order peaks allows for different layers of graphene to be distinguished [2], and an example of second order peaks used to distinguish graphene and bilayer graphene is shown in (f), the composite image of (b)-(d). 